\title{
Elevated $\mathrm{T} / \mathrm{E}_{2}$ Ratio Is Associated with an Increased Risk of Cerebrovascular Disease in Elderly Men
}

\author{
Yanping Gong ${ }^{19}$, Haiying Xiao ${ }^{19}$, Chunlin $\mathrm{Li}^{1 *}$, Jie Bai ${ }^{2}$, Xiaoling Cheng ${ }^{1}$, Mengmeng Jin ${ }^{1}$, Boruo Sun ${ }^{1}$, \\ Yanhui Lu' ${ }^{1}$, Yinghong Shao', Hui Tian ${ }^{1}$
}

1 Department of Geriatric Endocrinology, The Chinese People's Liberation Army General Hospital, Beijing, China, 2 Department of Geriatric Clinical Laboratory, The Chinese People's Liberation Army General Hospital, Beijing, China

\begin{abstract}
Objective: To investigate the relationship between sex hormones and the risk of vascular disease in elderly men and to evaluate the advantages and disadvantages of testosterone replacement.

Methods: A total of 337 men, aged 60 to 91 years, were enrolled in this single-center, cross-sectional study, and their sex hormone levels were assessed. Linear and logistic regression analyses were utilized to compare the sex hormone levels between patients with and without vascular disease. The nonparametric K-sample test was used for inter-group comparisons.

Results: Aging and abnormal metabolism were both significantly associated with an increased risk of vascular diseases and changes in sex hormone levels. Primary linear and logistic regression analyses showed no significant differences in sex hormone concentrations between patients with and without vascular diseases after adjusting for age. Logistic regression with abnormal metabolism as categorical variable showed that free testosterone (FT) and free estradiol $\left(\mathrm{FE}_{2}\right)$ had significant relationships with CEVD risk $(\mathrm{P}<0.05)$. In further regression with all metabolic continuous variables included, the testosterone/estradiol $\left(T / E_{2}\right)$ ratio replaced $F T$ and $F E_{2}(P<0.05)$. Trend line analyses showed that $T / E_{2}$ actually had a binomial linear correlation with the risk of cerebrovascular disease; its best protective effect occurred at values of $0.13-0.15$ with an OR value extremely close to those of $F T$ and $\mathrm{FE}_{2}(0.23$ vs. $0.24-0.25)$.

Conclusion: $\mathrm{T} / \mathrm{E}_{2}$ balance plays a key role in the relationship between sex hormones and the risk of cerebrovascular disease. The balance between $T$ and $E_{2}$ may be more important than their absolute quantities. Extremely low $T / E_{2}$ and inappropriately high $T / E_{2}$ ratio can both harm the brain blood vessels. Careful consideration should be given before beginning testosterone replacement treatment, and supplementing with estrogen seems to be a good way to protect blood vessels of the brain in elderly men.
\end{abstract}

Citation: Gong Y, Xiao H, Li C, Bai J, Cheng X, et al. (2013) Elevated T/E 2 Ratio Is Associated with an Increased Risk of Cerebrovascular Disease in Elderly Men. PLoS ONE 8(4): e61598. doi:10.1371/journal.pone.0061598

Editor: Jean-Marc A. Lobaccaro, Clermont Université, France

Received October 30, 2012; Accepted March 11, 2013; Published April 24, 2013

Copyright: (C) 2013 Gong et al. This is an open-access article distributed under the terms of the Creative Commons Attribution License, which permits unrestricted use, distribution, and reproduction in any medium, provided the original author and source are credited.

Funding: This work was supported by the grants from the National Natural Science Foundation of China (NFSC, No. 81173625, http://www.nsfc.gov.cn/Portal0/ default 152.htm) and MiaoPu Foundation (No. 12KMM33). The funders had no role in study design, data collection and analysis, decision to publish, or preparation of the manuscript.

Competing Interests: The authors have declared that no competing interests exist.

* E-mail: Icl301@sina.cn

9 These authors contributed equally to this work.

\section{Introduction}

The vasculature is a target tissue for sex steroid hormones. Estrogen and Testosterone (TT) have become research hotspots in recent years [1-3]. The relationship between TT and vascular disease has been the subject of much research. However, there is intense debate regarding the role of TT in vascular function and cardiovascular disease (CVD) [3]. Low TT is associated with the progression of atherosclerosis, the production of proinflammatory cytokines, increased arterial thickness, and increased glucose, total cholesterol and low-density lipoprotein, all of which contribute to CVD $[4,5]$. However, high TT has been associated with sudden cardiac death, liver disease and suicidal behavior [3,6]. Being male is a risk factor for CVD, as males have an earlier occurrence and higher prevalence of many cardiovascular and metabolic-related diseases [7].

In addition to TT, estrogen is significantly associated with the risk of vascular disease. Menopausal women have much lower incidences of heart and renal disease compared with men of the same age, but this sex difference in favor of women gradually disappears after menopause; indeed, the cardiovascular risk becomes even higher in older women [2,7]. The recent Nurse's Health Study [8], WISE Study [9], and the Women's Health Initiative [10] have demonstrated that early menopause in young women due to ovarian dysfunction or bilateral oophorectomy is associated with an increased risk of CVD compared with women exhibiting normal endogenous estrogen levels. Endogenous estrogen may be cardioprotective in men as well $[11,12]$. In 
men, significant amounts of estrogen can be produced by the enzyme aromatase, which converts C19 androgenic steroids into $17 \beta$-estradiol $\left(\mathrm{E}_{2}\right)$. In healthy young men, aromatase inhibition lowers plasma $\mathrm{E}_{2}$, and this decrease is associated with decreased flow-mediated dilatation of the brachial artery [13].

In contrast to women, whose estrogen levels drop precipitously at menopause, men of all ethnicities undergo a gradual decline in TT. Up to $30 \%$ of men over 60 years of age have TT levels that are below the normal range for young men; a $0.8 \%$ per year decrease in total TT and a $2 \%$ per year decrease in bioavailable TT was reported in a cross-sectional study of elderly men [14,15]. Elderly men, who suffer from many aging symptoms, such as fatigue and loss of muscle mass, could benefit greatly from testosterone replacement. Although much is known about the effects of hormones on blood vessels, there is still much to be discovered. It is necessary to determine the side effects of hormones on vessels. We designed this study to investigate the relationship between the changes in male sex hormone levels and the risk of vascular disease in elderly men.

\section{Methods}

\section{Study population}

This single-center, cross-sectional study enrolled men above 60 years of age. We screened 1920 men who were between 60 and 91 years of age who had routine physical examinations at the Chinese People's Liberation Army General Hospital between May and July 2011. Potential subjects were from all 16 districts and both counties of Beijing. After excluding those who had a malignancy or a disease that was treated using TT or corticosteroids, 508 elderly men (26.5\%) whose endogenous sex hormone levels had been determined remained as potential subjects. Among these potential subjects, the following patients were excluded: 90 who did not undergo biochemical examination, 79 who suffered from kidney dysfunction $(\mathrm{Cr}>113 \mu \mathrm{mol} / \mathrm{L}), 1$ who was diagnosed with hyperparathyroidism and 1 who had moderate anemia. No patient was excluded based on a diagnosis of liver dysfunction (alanine aminotransferase or aspartate aminotransferase 3 times above the normal level). Thus, 337 subjects (69.4\% from 558 ) were included in the analyses. The diagnosis of coronary heart disease or cerebrovascular disease was confirmed by reviewing each patient's medical record. The coronary heart diseases included angina, silent (asymptomatic) myocardial ischemia and myocardial infarction. The diagnoses of cerebrovascular disease included transient cerebral ischemia, vertebrobasilar insufficiency, cerebral thrombosis, cerebral infarction and cerebral hemorrhage.

\section{Clinical parameters}

After the patient fasted for at least 8 hours overnight, baseline parameters were obtained by trained physicians the next morning between 6:00 and 8:00. The recorded baseline parameters included height, weight, BMI $\left(\mathrm{kg} / \mathrm{m}^{2}\right)$, age, sex, medical history, medication use and relevant diseases. Blood pressure (BP) was measured twice at the right brachial artery with the patient in the sitting position after a 5 minutes rest using the semi-automatic oscillometric method.

\section{Sex hormone measurements}

A fasting venous blood sample was obtained in the morning between 6:00 and 8:00 after an overnight fast. A postprandial venous blood sample was extracted two hours after eating $100 \mathrm{~g}$ of carbohydrates or drinking liquid containing $75 \mathrm{~g}$ of glucose. The blood sample was immediately centrifuged for $15 \mathrm{~min}$ at $4^{\circ} \mathrm{C}$, and the platelet-free serum and plasma were stored at $-20^{\circ} \mathrm{C}$. All of the samples were tested within 1 week. Total TT, $\mathrm{E}_{2}$, sex hormone binding globulin (SHBG), follicle-stimulating hormone (FSH) and luteinizing hormone $(\mathrm{LH})$ were measured using an electrochemiluminescence system automatically (Roche Cobas C6000, Roche Diagnostics GmbH, Mannheim, Germany). Free testosterone (FT) and bioactive testosterone (BT) were calculated using Vermeulen's formula, free estradiol $\left(\mathrm{FE}_{2}\right)$ was calculated according to the formula reported by Sabina Rinald et al [16], and the $\mathrm{T} / \mathrm{E}_{2}$ and $\mathrm{E}_{2} / \mathrm{SHBG}$ ratios were also calculated. The FT index (FTI) was calculated as FTI $=\mathrm{T} / \mathrm{SHBG}$, and the testosterone secretion index $(\mathrm{TSI})$ was calculated as TSI $=\mathrm{T} / \mathrm{LH}$.

\section{Other laboratory measurements}

Biochemical indices were determined using serum samples that were collected after fasting, except for the postprandial blood glucose (PBG) measurement, which required postprandial serum samples. An automated enzymatic procedure (Cobas E601, Roche) was used to determine the fasting blood glucose (FBG) and PBG (Roche). High-density lipoprotein (HDL) and lowdensity lipoprotein (LDL) cholesterol, triglycerides (TG), serum total cholesterol (TC), uric acid (UA), blood urea nitrogen (BUN), creatinine $(\mathrm{Cr})$, glutamic-pyruvic transaminase (ALT) and glutamic-oxaloacetic transaminase (AST) were similarly measured. Diagnoses were defined according to the case records at the corresponding medical institution, if treatments or medications were utilized. New diagnoses were made if abnormal results were obtained, as described below. Diabetes mellitus was defined as FBG $>6.9 \mathrm{mmol} / \mathrm{L}$ and/or $\mathrm{PBG}>11.0 \mathrm{mmol} / \mathrm{L}$ and/or treatment with insulin or oral hypoglycemic agents. Impaired glucose tolerance (IGT) was defined as $\mathrm{FBG} \leq 6.9 \mathrm{mmol} / \mathrm{L}$ and $7.8 \mathrm{mmol} / \mathrm{L}<\mathrm{PBG} \leq 11.0 \mathrm{mmol} / \mathrm{L}$. Impaired fasting glucose (IFG) was defined as $6.1 \mathrm{mmol} / \mathrm{L}<\mathrm{FBG} \leq 6.9 \mathrm{mmol} / \mathrm{L}$ and $\mathrm{PBG} \leq 7.8 \mathrm{mmol} / \mathrm{L}$. Impaired glucose regulation (IGR) was defined as the combination of IGT and IFG. Hypertension was defined as systolic BP >140 mmHg and/or diastolic BP $>90 \mathrm{mmHg}$ and/or the use of anti-hypertensive medication. A patient was considered positive for hyperlipidemia if any lipid test result was abnormal (reference ranges defined by the Biochemistry Department of the Chinese People's Liberation Army General Hospital as TG $>5.7 \mathrm{mmol} / \mathrm{L}$, TG $>1.7 \mathrm{mmol} / \mathrm{L}$, HDL $<1.6 \mathrm{mmol} / \mathrm{L}$ and $\mathrm{LDL}>3.4 \mathrm{mmol} / \mathrm{L}$ ) and/or if the patient was taking any lipid-lowering medications. Hyperuricemia was defined as uric acid $>420 \mu \mathrm{mol} / \mathrm{L}$ and/or if corresponding medications were used. A patient was determined to be overweight with a BMI $>24 \mathrm{~kg} / \mathrm{m}^{2}$ and obese with a BMI $>28 \mathrm{~kg} / \mathrm{m}^{2}$. Diagnostic criteria for metabolic syndrome proposed by the Chinese Diabetes Society (CDS) were used (see Figure S1).

\section{Statistical analyses}

SPSS 17.0 (SPSS Inc, Chicago, IL, USA) was used for both data management and analyses. Continuous variables were presented as the mean $\pm \mathrm{SD}$. The distribution of data was evaluated before analyses. FSH, LH, E/SHBG, PROG and TSI did not have a normal distribution, exponential distribution or Poisson distribution. Therefore, we categorized the patients by vascular disease situation: cardiovascular disease only (CAVD), cerebrovascular disease only (CEVD), both type of vascular disease (TVD) and controls. After this categorization, only LH, FSH, and PROG did not have a normal distribution. Multiple-factor linear regression was used to compare normally distributed data, and nonparametric $\mathrm{K}$-sample tests were used to compare non-normally distributed data. Age, smoke, all sex hormones, BMI, BP (SBP, DBP), plasma glucose (FBG, PBG), insulin resistance (insulin, $\mathrm{C}$ peptide and homeostasis model assessment-estimated insulin resistance 
(HOMA-IR)), plasma lipids (TC, LDL, HDL and TG), plasma uric acid, and abnormal metabolism (MS and number of abnormal metabolic test results) were included in the stepwise regression analyses of vascular diseases. Index and log conversion of LH, FSH, PROG did not change the explanation effect of the regression equation. Classifying variables were reported as percentages. A $\chi^{2}$ test was conducted for two-group comparisons. Nonparametric K-sample tests were used for inter-group comparisons. Trend line analysis was used to analyze the tendency of sex hormone levels to increase with age. The $\mathrm{R}^{2}$ value was used to evaluate the effectiveness of the trend line. For these analyses, $\mathrm{P}<0.05$ was considered statistically significant. The 95\% confidence interval (95\% CI) was calculated to describe the magnitude of associations.

\section{Results}

\section{Vascular disease in this population}

A total of 337 subjects qualified for this study. The age ranged from 60 to 91 years. Included in the cohort were 101 (29.97\%) and $31(9.20 \%)$ subjects with CAVD and CEVD, respectively. There were $54(16.02 \%)$ subjects with both vascular diseases (TVD), which meant 186 (55.19\%) subjects had either type of the vascular diseases (EVD). The age distribution of the patients with vascular disease is depicted in Figure 1. The morbidity of cardiovascular disease, cerebrovascular disease and all vascular disease all increased with age $(\mathrm{P}<0.01)$.

\section{Sex hormone levels in this population}

The sex hormone levels of this population are presented in Table 1, and the sex hormone levels categorized by age group are illustrated in Figure 2. Serum FSH, LH, SHBG, FT and T/E ratio increased with age, but the others declined. FSH, LH, SHBG, FT, FTI, TSI, $\mathrm{FE}_{2}, \mathrm{E}_{2} / \mathrm{SHBG}$ ratio and PROG were linearly correlated with age, with $\mathrm{R}^{2}$ values of the linear equation of $0.92,0.94,0.97,0.88,0.99,0.92,0.85,0.96$ and 0.90 , respectively. FSH, LH, SHBG, FTI, TSI, $\mathrm{FE}_{2}$, and $\mathrm{E}_{2} / \mathrm{SHBG}$ changed significantly with age $(P<0.01)$.

\section{$\square$ control $\square$ CEVD only $\square$ CVD only $\square$ both}

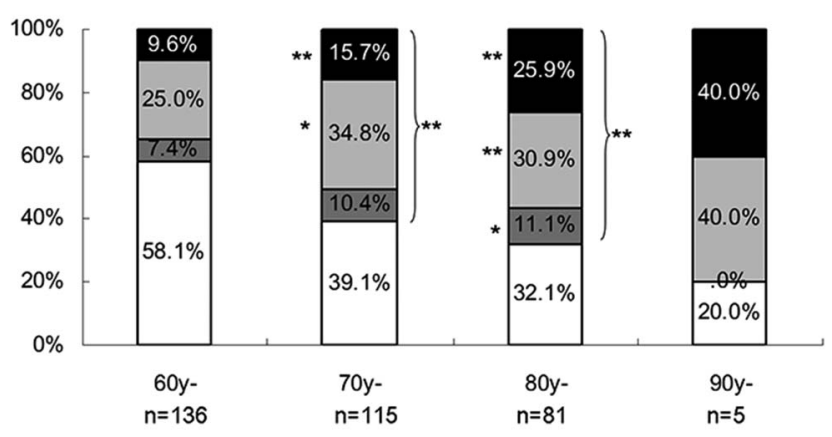

Figure 1. Age distribution of vascular diseases. The age range of the 60-year-old group was 60-69 years, of the 70-year-old age group was $70-79$ years, of the 80 -year-old group was $80-89$ years and of the 90-year-old group was 90-91 years. The morbidity of CEVD, CAVD, TVD and EVD are shown as a percentage of each age group. ${ }^{*} P<0.05$, ** $\mathrm{P}<0.01$ versus the morbidity of vascular diseases in the 60 -year-old group.

doi:10.1371/journal.pone.0061598.g001

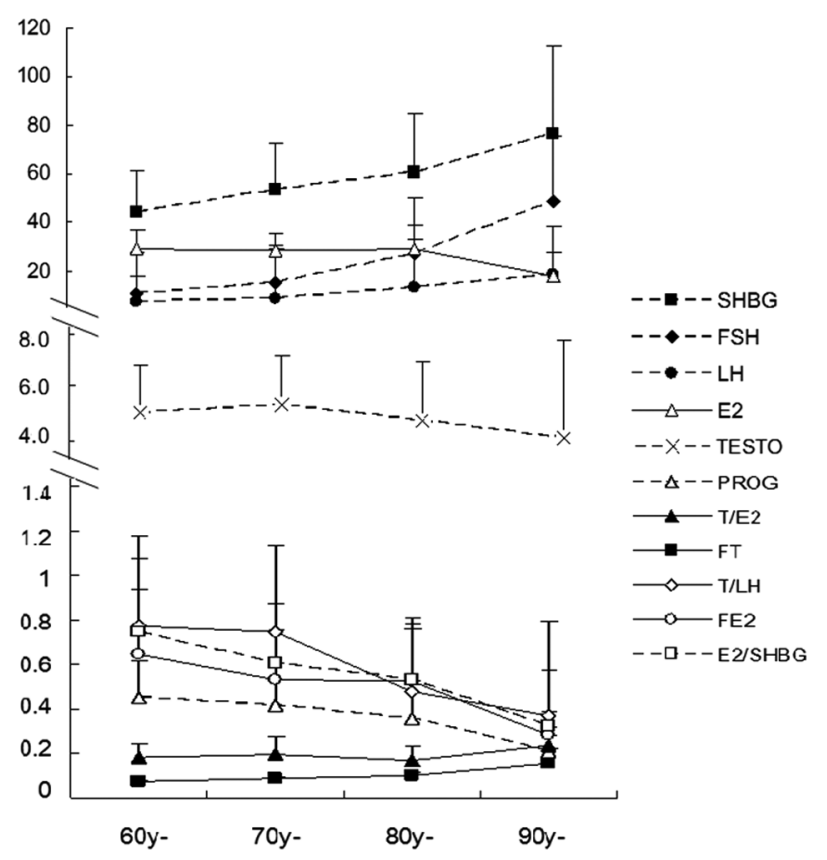

Figure 2. Changes in sex hormones by age category. The mean $\pm \mathrm{SD} T \mathrm{TT}(\mathrm{nmol} / \mathrm{L}), \mathrm{FT}(\mathrm{nmol} / \mathrm{L}), \mathrm{SHBG}(\mathrm{nmol} / \mathrm{L}), \mathrm{E}_{2}(\mathrm{pmol} / \mathrm{L}), \mathrm{FSH}(\mathrm{IU} / \mathrm{L})$, $\mathrm{LH}(\mathrm{IU} / \mathrm{L})$, PROG (nmol/L), TSI (nmol/IU), FTI, T/E $\mathrm{E}_{2}$ and $\mathrm{E}_{2} / \mathrm{SHBG}$ grouped by age are shown by curves. The age categories are the same as in Fig. 1. SHBG, $F T$ and $T / E_{2}$ increased with age (shown by filled symbols), but the others declined (shown with hollow symbols). FSH, LH, SHBG, $\mathrm{FT}, \mathrm{FTI}, \mathrm{TSI}, \mathrm{FE}_{2}, \mathrm{E}_{2} / \mathrm{SHBG}$ and PROG had a linear relationship with age, and the $R^{2}$ values of their linear equations were $0.92,0.94,0.97,0.88$, $0.99,0.92,0.85,0.96$ and 0.90 . Significant differences between age groups $(P<0.01)$ were observed in $\mathrm{FSH}, \mathrm{LH}, \mathrm{SHBG}, \mathrm{FTI}, \mathrm{TSI}, \mathrm{FE}_{2}$, and $\mathrm{E}_{2} /$ SHBG.

doi:10.1371/journal.pone.0061598.g002

Table 1. Characteristics of the sex hormones in the study population.

\begin{tabular}{|c|c|c|c|}
\hline & $\mathbf{n}$ & Mean \pm SD & Range \\
\hline FSH (IU/L) & 336 & $16.89 \pm 16.89$ & $0.10-119.40$ \\
\hline LH (IU/L) & 336 & $9.42 \pm 6.29$ & $0.10-45.69$ \\
\hline $\mathrm{E}_{2}(\mathrm{pmol} / \mathrm{L})$ & 335 & $28.51 \pm 8.6$ & $5.00-55.90$ \\
\hline$E_{2} / S H B G$ & 335 & $0.64 \pm 0.00$ & $0.14-1.76$ \\
\hline $\mathrm{FE}_{2}$ & 335 & $0.59 \pm 0.27$ & $0.17-1.88$ \\
\hline PROG (nmol/L) & 336 & $0.41 \pm 0.18$ & $0.00-1.09$ \\
\hline$\pi(\mathrm{nmol} / \mathrm{L})$ & 337 & $5.04 \pm 1.93$ & $0.03-11.16$ \\
\hline $\mathrm{T} / \mathrm{E}_{2}$ & 335 & $0.18 \pm 0.00$ & $0.00-0.44$ \\
\hline FTI & 337 & $0.10 \pm 0.03$ & $0.00-0.22$ \\
\hline TSI (nmol/IU) & 336 & $0.69 \pm 0.39$ & $0.00-2.26$ \\
\hline $\mathrm{FT}(\mathrm{nmol} / \mathrm{L})$ & 337 & $0.22 \pm 0.08$ & $0.00-0.67$ \\
\hline BT (nmol/L) & 337 & $5.80 \pm 1.91$ & $0.01-11.1$ \\
\hline PRL $(\mu \mathrm{g} / \mathrm{L})$ & 336 & $28.51 \pm 8.60$ & $0.00-55.90$ \\
\hline SHBG $(\mathrm{nmol} / \mathrm{L})$ & 337 & $51.82 \pm 21.42$ & $11.38-172.50$ \\
\hline
\end{tabular}

doi:10.1371/journal.pone.0061598.t001 


\section{The association between vascular disease and sex hormone levels}

As shown in Table 2, plasma TT, BT, FTI, TSI, $\mathrm{E}_{2}, \mathrm{E}_{2} / \mathrm{SHBG}$ and PCG were lower in patients with vascular disease compared with the control group, while LH, FSH and SHBG were higher in the vascular disease groups. The changes in the above sex hormones in CEVD were more obvious than in CAVD. LH, FSH, SHBG, FTI and TSI were significantly different between vascular disease groups. After adjustment for age and smoke, the gaps in these sex hormones between controls and vascular disease patients decreased, and all the significant differences disappeared. Logistic analyses showed the same results with age and smoke as covariant.

Considering that abnormal metabolism also had a close correlation with vascular disease, we performed logistic regression with metabolic indexes as covariates (see Table 3). In regression with categorical variables of abnormal metabolism as covariates, PROG, FT, $\mathrm{FE}_{2}$ and MetS showed close correlations with cerebrovascular disease. The value of $\exp (\mathbf{B})$ for these four parameters was $1.00,>1000,0$ and 4.24 , respectively. In the regression including continuous variables of metabolic parameters as covariates (see Table 3), the significant correlations of FT and $\mathrm{FE}_{2}$ with cerebrovascular disease disappeared, and instead, elevated $\mathrm{T} / \mathrm{E}_{2}$ ratio, $\mathrm{LH}$ and $\mathrm{C}$-peptide were correlated with increased incidence of cerebrovascular disease $(\mathrm{P}<0.05)$, with an $\exp (\mathrm{B})$ value of $>1000,1.1$ and 4.09, respectively.

No correlations were found between sex hormones and CAVD, TVD, or EVD. Instead, abnormal glucose level, hyperlipidemia, BMI and MetS showed close correlations with these 3 vascular diseases.

\section{Trend line of $\mathrm{FT}, \mathrm{FE}_{2}$, and $\mathrm{T} / \mathrm{E}_{2}$ in elder men}

ROC curves were first used to explore the possible cut-off values of $\mathrm{FT}, \mathrm{FE}_{2}$ and $\mathrm{T} / \mathrm{E}_{2}$, but the areas under the curve were not ideal (the highest was 0.59 , from $\mathrm{T} / \mathrm{E}_{2}$, see Figure $3 \mathrm{~A}$ ). This combined with the fact that the $95 \% \mathrm{CI}$ of $\mathrm{FT}, \mathrm{FE}_{2}$ and $\mathrm{T} / \mathrm{E}_{2}$ were not present in a relatively confined areas, we speculated that the relationships of FT, T/E $\mathrm{E}_{2}$ and $\mathrm{CEVD}$ were not simple linear relationships. We analyzed these parameters based on quartiles, and binomial linear relationships of GEVD with these three sex hormones were found. Positive trends of FT and $\mathrm{T} / \mathrm{E}_{2}$ and a negative trend of $\mathrm{FE}_{2}$ were detected, which were consistent with the values of $\exp (\mathrm{B})$ from logistic regression. FT and $\mathrm{T} / \mathrm{E}_{2}$ in quartile $4\left(Q^{4}\right)$ were associated with a higher risk of a CEVD event $\left(\mathrm{OR}=2.23\right.$ and $2.36, \mathrm{P}=0.05$ and 0.03 , respectively), and $\mathrm{FE}_{2}$ in $\mathrm{Q} 4$ was associated with a lower risk of a CEVD event $(\mathrm{OR}=0.29$, $\mathrm{P}=0.04$ ), compared with the values in Q1-3 (Fig. 3B-D).

We further divided quartiles $2-4$ of $\mathrm{FE}_{2}, \mathrm{FT}$ and $\mathrm{T} / \mathrm{E}_{2}$ into 8,8 and 6 groups by increments of $0.05 \mathrm{pmol} / \mathrm{L}, 0.02 \mathrm{nmol} / \mathrm{L}$ and 0.01 units, respectively, creating subgroups of 10-20 people each. The trend lines and equations are shown in Fig. 3E-G. Calculated from the formulas, the best protective effect was observed when $\mathrm{FE}_{2}$ increased to more than $0.65-0.75 \mathrm{pmol} / \mathrm{L}$, FT remained at $0.16-0.18 \mathrm{nmol} / \mathrm{L}$, and $\mathrm{T} / \mathrm{E}_{2}$ remained at $0.13-0.15$. The $\mathrm{OR}$ values of these areaes compared with the highest risk areas of $\mathrm{FE}_{2}$, $\mathrm{FT}$ and $\mathrm{T} / \mathrm{E}_{2}$ were extremely close to $(0.24,0.25$ and 0.23 , respectively).

\section{Discussion}

In this study population, age is an important factor that influences both vascular diseases and sex hormones: the incidence of vascular disease increases with age, and the levels of sex hormones vary with age. However, age is not the only factor that influenced sex hormones. Our earlier study confirmed that

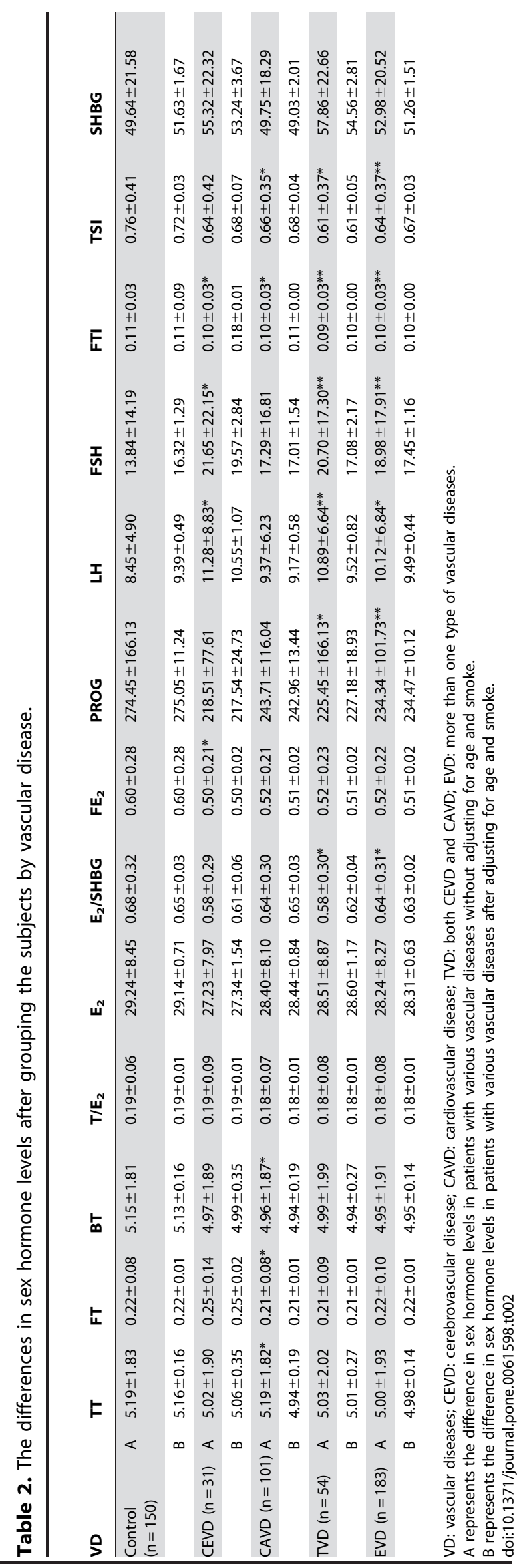

PLOS ONE | www.plosone.org 
Table 3. Logistic regression analyses of cerebrovascular disease.

\begin{tabular}{|c|c|c|c|c|c|c|c|c|c|}
\hline & PROG & $\mathbf{F T}$ & $\mathrm{FE}_{2}$ & $E_{2} / S H B G$ & $T / E_{2}$ & LH & Age & MetS & C-peptide \\
\hline \multicolumn{10}{|l|}{ model 1} \\
\hline sig & 0.05 & 0.05 & & & & & & & \\
\hline $\exp (B)$ & 1 & 75.71 & & & & & & & \\
\hline 95\%C.I. & $0.99-1.02$ & $1.10->1000$ & & & & & & & \\
\hline \multicolumn{10}{|l|}{ model 2} \\
\hline sig & 0.06 & 0.09 & & & & & 0.05 & & \\
\hline $\exp (B)$ & 1.0 & 45.16 & & & & & 1.058 & & \\
\hline 95\%C.I. & $0.99-1.00$ & $0.58-3494$ & & & & & $1.01-1.12$ & & \\
\hline \multicolumn{10}{|l|}{ model 3} \\
\hline sig & 0.05 & 0.02 & 0.04 & 0.58 & & 0.11 & 0.43 & 0.022 & \\
\hline $\exp (B)$ & 1 & $>1000$ & 0.00 & 166.65 & & 1.07 & 1.03 & 4.24 & \\
\hline 95\%C.I. & $0.99-1.00$ & $4.17->1000$ & $0.00-0.69$ & $0.84->1000$ & & $0.99-1.15$ & $0.97-1.11$ & $1.23-14.67$ & \\
\hline \multicolumn{10}{|l|}{ model 4} \\
\hline sig & 0.07 & 0.41 & 0.106 & & 0.05 & 0.03 & & & 0.03 \\
\hline $\exp (B)$ & 1 & $>1000$ & 0.21 & & $>1000$ & 1.10 & & & 4.09 \\
\hline 95\%C.I. & $0.99-1.00$ & $<0.01->1000$ & $0.16-1.52$ & & $0.95->1000$ & $1.00-1.28$ & & & $1.14-14.68$ \\
\hline
\end{tabular}

Model 1: all sex hormones were included in the regression equation.

Model 2: all sex hormones and age were included in the regression equation.

Model 3: all sex hormones, age, all metabolic categorical variables and hepatic/renal function were included in the regression equation.

Model 4: all sex hormones, age, all metabolic continuous variables and hepatic/renal function were included in the regression equation.

doi:10.1371/journal.pone.0061598.t003

abnormal metabolism can change the sex hormone levels in elder men [17]. FT and $\mathrm{FE}_{2}$ were correlated with GEVD in linear regressions with categorical variables of abnormal metabolism included, and the effect of elevated $\mathrm{T} / \mathrm{E}_{2}$ was highlighted in linear regressions with continuous variables included, though no significant differences in sex hormones could be found with only age adjustment in linear and logistic regression analyses.

The changes in testosterone and estrogen levels with increasing age are different between men and women $[18,19]$. Testosterone peaks in men are in young adulthood. Starting around age 30, however, testosterone levels gradually decline. One reason for this change is that testosterone is more likely to be converted to estradiol by aromatase in aging men. Furthermore, as the ratio of testosterone to estrogen begins to shift, the chance that estrogen will bind to androgen receptors (normally used by testosterone) increases. The body then responds by producing less testosterone, which shifts the balance even more.

Previous cohort studies of the association between serum testosterone and $\mathrm{CV}$ events have found contradictory results. Large population-based prospective studies reported an inverse association (Rancho Bernardo [20], European Investigation into Cancer-Norfolk [21]), no association (Massachusetts Male Aging Study [15], National Health And Nutrition Examination Survey [22]), or a positive association (Vlachopoulos [6,15], Wittnich [23]) between total testosterone and CV disease mortality [15,24]. Our study showed that the trend line of CEVD and finely grouped FT values was defined by a binomial line, and maintaining a proper range of FT $(0.16-0.18 \mathrm{pmol} / \mathrm{L})$ might be helpful to prevent vascular diseases. Considering that this range of FT was lower than that observed in most of our patients, higher FT would usually have positive relationship with increasing risk of GEVD. Subjects in the highest quartile of FT had a 2-fold higher risk of CEVD compared with the lowest quartile. At the same time, the risk of cerebrovascular disease could be increased by androgen supplementation, so careful consideration should be given before beginning treatment.

Estrogen can also offer vascular protection [2,12,13,18]. Our study confirmed that higher estrogen significantly decreased the VECD incidence, which was only $29 \%$ in the highest $\mathrm{FE}_{2}$ quartile compared with the lowest quartile. At the same time, our data suggest that $7.5 \mathrm{pmol} / \mathrm{L}$ of free estrogen is the cut-off point above which the risk of CEVD decreases $86 \%$ compared to the risk of the highest-risk group. Supplementation with estrogen therefore seems a good way to protect the blood vessels of the brain in elderly men. However, for elderly men whose estrogen is already extremely high, simple estrogen replacement might not be appropriate. A recent study [25] measured blood estradiol in 501 men with chronic heart failure and found that men in the highest quintile of estradiol (serum estradiol more than $37.40 \mathrm{pg} / \mathrm{mL}$ or $137.26 \mathrm{pmol} / \mathrm{L}$ ) had an increased death rate. The authors recommended that in aging males, estradiol should be kept under $30 \mathrm{pg} / \mathrm{mL}(110.1 \mathrm{pmol} / \mathrm{L})$. Neither $\mathrm{E}_{2}$ nor $\mathrm{FE}_{2}$ in our study reached such a high level, so we did not find an upper recommended limit of $\mathrm{FE}_{2}$. However, when metabolic abnormalities were set as continuous variables in the logistic regression analyses, the effect of the $\mathrm{T} / \mathrm{E}_{2}$ ratio gained significance. This meant $\mathrm{FE}_{2}$ could not increase indefinitely along with lower $\mathrm{T}$ in elderly men, and the upper the limit of $\mathrm{FE}_{2}$ depended on the ratio of $\mathrm{T} / \mathrm{E}_{2}$.

Furthermore, in regression with all metabolic continuous variables included, $\mathrm{T} / \mathrm{E}_{2}$ took the place of $\mathrm{FT}$ and $\mathrm{FE}_{2}$, with the best CEVD protection having at $\mathrm{T} / \mathrm{E}_{2}$ ratio of $0.13-0.15$, whose $\mathrm{OR}$ value was extremely similar to that of $\mathrm{FT}$ and $\mathrm{FE}_{2}(0.23$ vs. $0.24-0.25)$. These values indicated that the $\mathrm{T} / \mathrm{E}_{2}$ ratio might be the most relevant sex hormone parameter in CEVD. On the other hand, the significant effect of $\mathrm{T} / \mathrm{E}_{2}$ also reflected the fact that increased SHBG, increased aromatase inhibition, higher TT, lower $\mathrm{E}_{2}$ and other factors that change the $\mathrm{TT} / \mathrm{E}_{2}$ ratio might all 

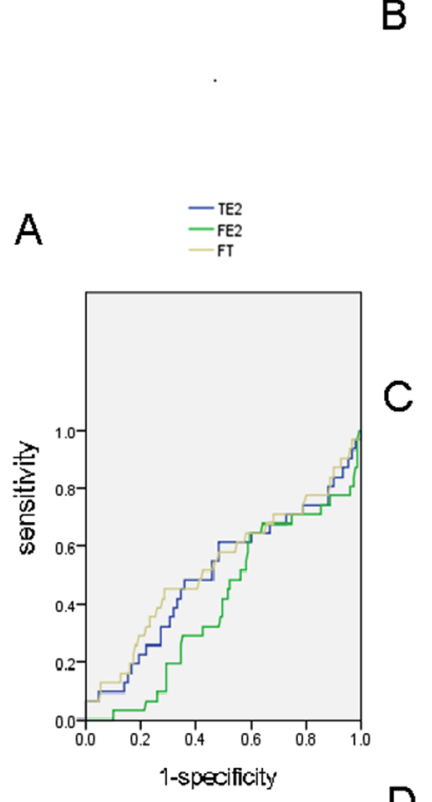

$\mathrm{D}$

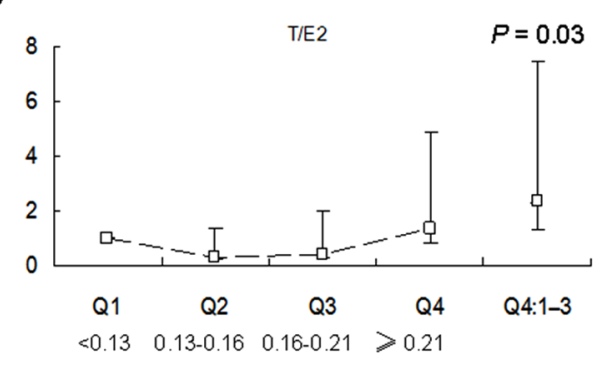

$\mathrm{E}$

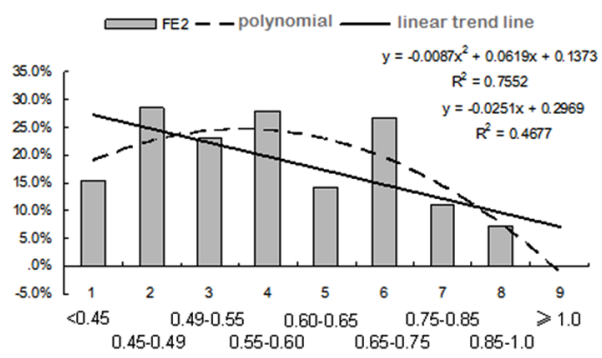

F

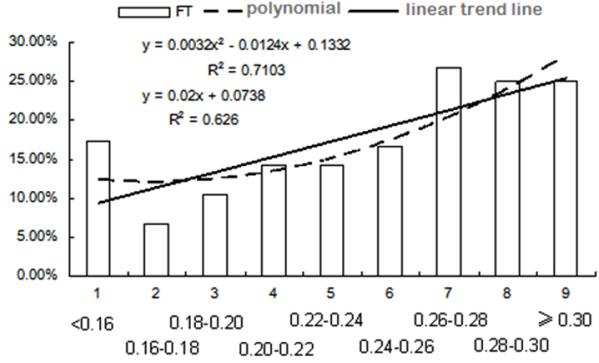

G

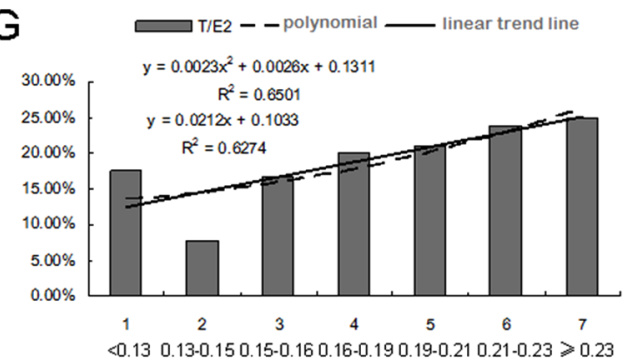

Figure 3. Trend lines of $F T, F E_{2}$, and $T / E_{2}$ and CEVD in elderly men. ROC curves of $F T, F E_{2}$ and $T / E_{2}$ are shown in $3 A$. Using men in quartile 1 (Q1) as a reference, the risk of CEVD of Q2, Q3, and Q4 are shown in Fig. 3B-D. "Q4:Q1-3" means the risk of CEVD of Q4 compared to the pool of Q13. We divided quartiles $2-4$ of $\mathrm{FE}_{2}, \mathrm{FT}$ and $\mathrm{T} / \mathrm{E}_{2}$ into 8,8 and 6 groups by increments of $0.05 \mathrm{pmol} / \mathrm{L}, 0.02 \mathrm{nmol} / \mathrm{L}$ and 0.01 units, respectively $(10$ to 20 people per group). The trend lines and equations are shown in Fig. $3 \mathrm{E}-\mathrm{G}$. doi:10.1371/journal.pone.0061598.g003

contribute to the increased CEVD incidence. The balance between these two hormones may be more important than their absolute quantities, which partly explains why studies focusing on androgens or estrogens alone have produced inconclusive results. For example, postmenopausal women, young men, and polycystic ovary syndrome patients, who have low $\mathrm{E}_{2}$ and/or high TT, all have higher risk of vascular diseases than young control women [2]. Therefore, if we want to improve muscle strength or bone BMD in elderly men by testosterone supplementation, a combination of appropriate doses of testosterone and estrogen might be necessary. The proposed balance of $\mathrm{T} / \mathrm{E}_{2}$ in elderly men is shown in Fig. 4.

Surprisingly, CEVD had a positive correlation with increased $\mathrm{FT}$, and $\mathrm{FT}, \mathrm{FE}_{2}$ and $\mathrm{T} / \mathrm{E}_{2}$ all had ideal equations in logistic regression. We believe that including full and comprehensive metabolic indexes and sex hormone indexes in this study was the key difference between our study and other studies. For example, when $\mathrm{F} / \mathrm{E}_{2}$ was included in the regression, the effect of SHBG disappeared (data not shown). Similarly, the relationships between sex hormones and CEVD were detected only after indexes of metabolism were included, and whether categorical variables or continuous variables were included in the regression affected the results of our analyses. Vlachopoulos et al. [6] also found in a prospective study that the correlation of low plasma $\mathrm{T}$ with increased risk of cardiovascular disease in ANOVA was com-

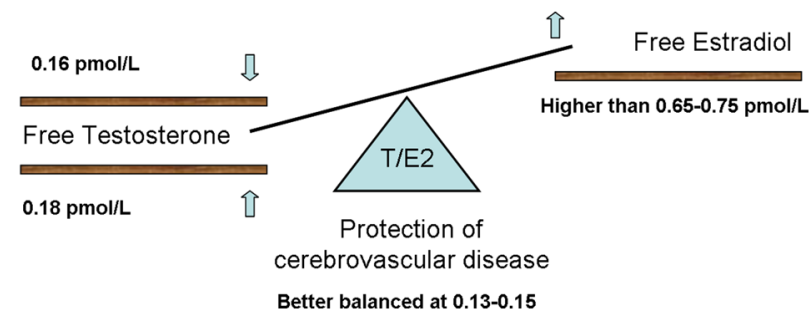

Figure 4. The balance of $T / E_{\mathbf{2}}$ in elder men. Better CEVD protection was observed when $\mathrm{FE}_{2}$ was higher than $7.5 \mathrm{pmol} / \mathrm{L}$. At the same time, the patient should be kept in the proper ranges of $F T$ and $T / E_{2}$. The balance between $T$ and $E_{2}$ may be more important than their absolute quantities.

doi:10.1371/journal.pone.0061598.g004

pletely reversed in Cox analyses when standard metabolic risk factors were included. We wonder whether analyses based on data with many confounding factors is one of the factors (other than the ratio of $\mathrm{T} / \mathrm{E}_{2}$ ) that have confounded the effects of sex hormones on vascular diseases in some former studies.

To put our findings in context, some limitations need to be addressed. Our study is cross-sectional, and the cross-sectional estimates of associations are not true measures of longitudinal 
changes. We have collected 2000 more subjects in routine physical examinations in 2012, and the 337 subjects of this study have been followed up to the greatest extent possible. An appropriately powered, placebo-controlled trial is being started to verify the possible protective effect of combined supplementation with testosterone and estrogen in elderly men, and the optimal $\mathrm{T} / \mathrm{E}_{2}$ ratio will also be evaluated.

\section{Conclusion}

Sex hormone levels were tightly correlated with the incidence of cerebrovascular disease in elderly men. Elevated FT levels and a higher $\mathrm{T} / \mathrm{E}_{2}$ ratio might increase the risk of cerebrovascular disease, while higher $\mathrm{FE}_{2}$ decreased this risk. Elevated $\mathrm{T} / \mathrm{E}_{2}$ was the key parameter in the relationship between sex hormones and the risk of cerebrovascular disease. The balance between $\mathrm{T}$ and $\mathrm{E}_{2}$ may be more important than their absolute quantities. Extremely low or high $\mathrm{T} / \mathrm{E}_{2}$ values will harm the brain blood vessels. Careful consideration should be given before beginning testosterone

\section{References}

1. Wu FC, Tajar A, Beynon JM, Pye SR, Silman AJ, et al. (2010) Identification of late-onset hypogonadism in middle-aged and elderly men. N Engl J Med 363: 123-135.

2. Yang XP, Reckelhoff JF (2011) Estrogen, hormonal replacement therapy and cardiovascular disease. Curr Opin Nephrol Hypertens 20: 133-138.

3. Sher L (2012) High and low testosterone levels may be associated with suicidal behavior in young and older men, respectively. Aust N Z J Psychiatry.

4. Francomano D, Bruzziches R, Natali M, Aversa A, Spera G (2010) Cardiovascular effect of testosterone replacement therapy in aging male. Acta Biomed 81 Suppl 1: 101-106.

5. Svartberg J, von Muhlen D, Mathiesen E, Joakimsen O, Bonaa KH, et al. (2006) Low testosterone levels are associated with carotid atherosclerosis in men. J Intern Med 259: 576-582.

6. Vlachopoulos C, Ioakeimidis N, Terentes-Printzios D, Aznaouridis K, Rokkas $\mathrm{K}$, et al. (2013) Plasma total testosterone and incident cardiovascular events in hypertensive patients. Am J Hypertens 26: 373-381.

7. Schierbeck LL, Rejnmark L, Tofteng CL, Stilgren L, Eiken P, et al. (2012) Effect of hormone replacement therapy on cardiovascular events in recently postmenopausal women: randomised trial. BMJ 345: e6409.

8. Brett KM (2005) Can hysterectomy be considered a risk factor for cardiovascular disease? Circulation 111: 1456-1458.

9. Gierach GL, Johnson BD, Bairey Merz CN, Kelsey SF, Bittner V, et al. (2006) Hypertension, menopause, and coronary artery disease risk in the Women's Ischemia Syndrome Evaluation (WISE) Study. J Am Coll Cardiol 47: S50-58.

10. Anderson GL, Limacher M, Assaf AR, Bassford T, Beresford SA, et al. (2004) Effects of conjugated equine estrogen in postmenopausal women with hysterectomy: the Women's Health Initiative randomized controlled trial. JAMA 291: 1701-1712.

11. Wenner MM, Stachenfeld N (2012) Blood pressure and water regulation: understanding sex hormone effects within and between men and women. J Physiol.

12. Gardner JD, Murray DB, Voloshenyuk TG, Brower GL, Bradley JM, et al. (2010) Estrogen attenuates chronic volume overload induced structural and functional remodeling in male rat hearts. Am J Physiol Heart Circ Physiol 298: H497-504.

13. Naessen T, Bergquist J, Lind L, Kushnir MM (2012) Higher endogenous estrogen levels in 70-year-old women and men: an endogenous response to counteract developing atherosclerosis? Menopause. replacement treatment, and supplementation with estrogen seems a good way to protect the blood vessels of the brain in elderly men.

\section{Supporting Information}

Figure S1 Diagnostic criteria for metabolic syndrome proposed by the Chinese Diabetes Society (GDS).

(TIF)

\section{Acknowledgments}

The authors are grateful to all participants for their full commitments and co-operation in the study.

\section{Author Contributions}

Conceived and designed the experiments: CLL YPG JB YHS HT. Performed the experiments: YPG HYX XLC BRS MMJ YHL. Analyzed the data: YPG HYX XLC BRS MMJ YHL. Contributed reagents/ materials/analysis tools: CLL JB HT. Wrote the paper: YPG CLL YHS.

14. Grossmann M (2010) Diagnosis and treatment of hypogonadism in older men: proceed with caution. Asian J Androl 12: 783-786.

15. Feldman HA, Longcope C, Derby CA, Johannes CB, Araujo AB, et al. (2002) Age trends in the level of serum testosterone and other hormones in middle-aged men: longitudinal results from the Massachusetts male aging study. J Clin Endocrinol Metab 87: 589-598.

16. Rinaldi S, Geay A, Dechaud H, Biessy C, Zeleniuch-Jacquotte A, et al. (2002) Validity of free testosterone and free estradiol determinations in serum samples from postmenopausal women by theoretical calculations. Cancer Epidemiol Biomarkers Prev 11: 1065-1071.

17. Gong Y, Xiao H, Bai J, Li C, Wen X, et al. (2013) Association between sex hormone levels and abnormal metabolism in a population of elderly Chinese men. Aging Male 16: 8-16.

18. Dai W, Li Y, Zheng H (2012) Estradiol/Testosterone Imbalance: Impact on Coronary Heart Disease Risk Factors in Postmenopausal Women. Cardiology 121: 249-254

19. Zheng HY, Li Y, Dai W, Wei CD, Sun KS, et al. (2012) Imbalance of testosterone/estradiol promotes male CHD development. Biomed Mater Eng 22: 179-185.

20. Laughlin GA, Barrett-Connor E, Bergstrom J (2008) Low serum testosterone and mortality in older men. J Clin Endocrinol Metab 93: 68-75.

21. Khaw KT, Dowsett M, Folkerd E, Bingham S, Wareham N, et al. (2007) Endogenous testosterone and mortality due to all causes, cardiovascular disease, and cancer in men: European prospective investigation into cancer in Norfolk (EPIC-Norfolk) Prospective Population Study. Circulation 116: 2694-2701.

22. Menke A, Guallar E, Rohrmann S, Nelson WG, Rifai N, et al. (2010) Sex steroid hormone concentrations and risk of death in US men. Am J Epidemiol 171: 583-592.

23. Wittnich C, Tan L, Wallen J, Belanger M (2013) Sex differences in myocardial metabolism and cardiac function: an emerging concept. Pflugers Arch.

24. Bhasin S, Cunningham GR, Hayes FJ, Matsumoto AM, Snyder PJ, et al. (2010) Testosterone therapy in men with androgen deficiency syndromes: an Endocrine Society clinical practice guideline. J Clin Endocrinol Metab 95: 2536-2559.

25. Jankowska EA, Rozentryt P, Ponikowska B, Hartmann O, KustrzyckaKratochwil D, et al. (2009) Circulating estradiol and mortality in men with systolic chronic heart failure. JAMA 301: 1892-1901. 